\title{
Colloidal Trajectories in Two-Dimensional Light-Induced Quasicrystals with 14-Fold Symmetry due to Phasonic Drifts
}

\author{
M. Martinsons*, M. SAndbrink And M. SchmiedeberG \\ Institut für Theoretische Physik 2: Weiche Materie, Heinrich-Heine-Universität Düsseldorf \\ 40225 Düsseldorf, Germany
}

\begin{abstract}
Quasicrystals are structures that are not periodic but possess a long range positional order. They can have any rotational symmetry including those that are forbidden in periodic crystals. The symmetry is classified by the point group and the rank $D$. In quasicrystals, phasons as additional hydrodynamic modes cause correlated rearrangements of the particles. The number of phasonic degrees of freedom depends on the rank. For colloidal quasicrystals that are induced by laser fields, specific phasonic displacements can be realized by changing the phases of the laser beams in a well-determined way. The arising trajectories of colloids in two-dimensional light-induced colloidal quasicrystals with rank $D=4$ have already been analyzed in detail. Here, we analyze the colloidal trajectories in two-dimensional quasicrystals with 14-fold symmetry having rank $D=6$. We observe complex paths of the colloids consisting of straight and winding lines as well as jumps.
\end{abstract}

DOI: $10.12693 /$ APhysPolA.126.568

PACS: 82.70.Dd, 61.44.Br

\section{Introduction}

Phasons are additional hydrodynamic modes of quasicrystals similar to conventional phononic excitations. In contrast to a periodic crystal, a quasicrystal of dimension $d$ can possess several $n$ relatively incommensurate length scales per direction leading to $D=n d$ hydrodynamic variables whose excitations do not cost free energy in the long wavelength limit. Let us note that there are also quasicrystals with different numbers of incommensurate length scales in different directions (see e.g. discussion in [1]). $D$ is termed the rank of the quasicrystal. These modes are divided into $d$ phononic ones which in the long wavelength limit correspond to translations of the atoms and $D-d$ phasonic ones which resemble complex correlated rearrangements of atoms. In particular, many other properties of phasons are still a main topic in research [2].

In statistical physics, colloidal particles are a wellknown model system to study phenomena like ordering, crystallization, and dynamics in external potentials [3]. In a laser field colloids are forced towards the areas of highest light intensity $[4,5]$. This allows the formation of complex structures [6]. For example, quasicrystalline ordering can be established by employing an interference pattern with quasicrystalline symmetry [7-18], these colloidal systems connect to the growing subject of soft matter quasicrystals (see, e.g., [19]). By tuning the laser phases appropriately it is possible to change the phasonic displacement of the system in a controlled way $[9,11,16]$. Thus, we can study the trajectories of the colloids that follow the maxima in the light field.

\footnotetext{
* corresponding author; e-mail:

Miriam.Martinsons@uni-duesseldorf.de
}

In recent works $[16,20]$, a method was presented to predict the trajectories of colloidal particles in a quasicrystalline laser field when a phasonic drift is applied, i.e., when the phasonic displacement changes at a constant rate in time. It was shown that every colloid can be mapped into characteristic areas of reduced phononic and phasonic displacement. Therefore, one only has to study the paths inside these areas to derive the behavior for the whole quasicrystal.

So far quasicrystalline interference patterns with 8-, 10- and 12-fold rotational symmetry were considered, which are all of rank $D=4$. Here, we show on the basis of the 14-fold symmetry how the method can be further extended to laser fields with rank $D=6$. Compared to the $D=4$ case, the additional phasonic modes lead to a more complex behavior and we identify several new types of colloidal trajectories.

In Sect. 2 we introduce the model system. The characteristic areas of reduced phononic and phasonic displacement are determined in Sect. 3. In Sect. 4 we study the behavior inside these areas to predict the colloidal trajectories in general. Selected examples are analyzed in detail. Finally, we conclude in Sect. 5 .

\section{Laser fields with quasicrystalline symmetry and phasonic displacements}

A light field acts on colloids like an external potential. The interference pattern created by 7 laser beams arranged as illustrated in Fig. 1a corrensponds to an external potential $[7,18,21]$ of the form

$$
V(\boldsymbol{r})=-\frac{V_{0}}{49} \sum_{j=0}^{6} \sum_{k=0}^{6} \cos \left(\left(\boldsymbol{G}_{j}-\boldsymbol{G}_{k}\right) \cdot \boldsymbol{r}+\phi_{j}-\phi_{k}\right),
$$

where $\boldsymbol{G}_{j}=\left(2 \pi / a_{\mathrm{V}} \cos (2 \pi j / 7), 2 \pi / a_{\mathrm{V}} \sin (2 \pi j / 7)\right)$ with $j=0, \ldots, 6$ are the wave vectors projected on the sample plane depicted in Fig. $1 \mathrm{~b} ; a_{\mathrm{V}}=2 \pi /\left|\boldsymbol{G}_{j}\right|$ denotes the 
length scale of the potential and $\phi_{j}$ the phases of the laser beams. The interference pattern exhibits 14-fold symmetry and is illustrated in Fig. 1c.

(a)

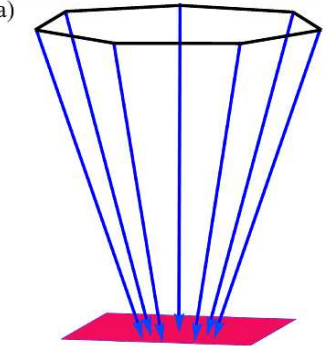

(c)

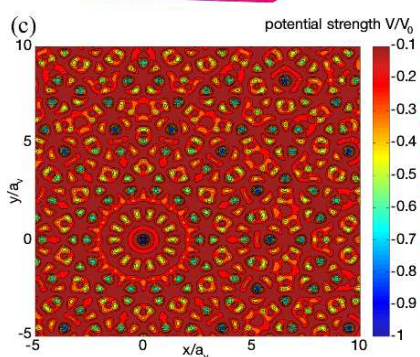

Fig. 1. (a) Setup of seven interfering laser beams. (b) Projection of the wave vectors on the sample plane. (c) Resulting interference pattern. High laser intensities corresponding to minima in the potential are depicted dark blue. Lower intensities appear in brighter red.

(d) Colloids in the minima of a potential with 14-fold symmetry.

Our parameterization of the phases is similar to [22] and [23]:

$$
\phi_{j}=\boldsymbol{G}_{j} \cdot \boldsymbol{u}+\boldsymbol{G}_{3 j \bmod 7} \cdot \boldsymbol{v}+\boldsymbol{G}_{5 j \bmod 7} \cdot \boldsymbol{w},
$$

where $\boldsymbol{u}=\left(u_{x}, u_{y}\right)$ denotes the phononic, $\boldsymbol{v}=\left(v_{x}, v_{y}\right)$ and $\boldsymbol{w}=\left(w_{x}, w_{y}\right)$ the phasonic displacements. Let us note that compared to a $D=4$ quasicrystal an additional phasonic displacement vector is required.

In the following, we consider the limit of large potential strengths or low temperatures $\left(V_{0} \gg k_{\mathrm{B}} T\right)$, such that all colloids are located in the minima of the potential (cf. snapshot shown in Fig. 1d).

\section{Characteristic displacements and characteristic areas}

In a periodic crystal, we can deduce many properties from a single unit cell $[24,25]$. In a quasicrystal, we can map all particle positions onto particles inside characteristic areas of small phononic and phasonic displacements $[16,20]$. To determine the characteristic areas, we first calculate phononic and phasonic displacements $\Delta \boldsymbol{u}, \Delta \boldsymbol{v}$ and $\Delta \boldsymbol{w}$ that change the differences between the phases $\phi_{j}-\phi_{k}$ in Eq. (1) only by integer multiples of $2 \pi$. We solve $V \boldsymbol{v}+\Delta \boldsymbol{v}, \boldsymbol{w}+\Delta \boldsymbol{w}(\boldsymbol{r}+\Delta \boldsymbol{u})=V \boldsymbol{v}, \boldsymbol{w}(\boldsymbol{r})$ where $V \boldsymbol{v}, \boldsymbol{w}(\boldsymbol{r})$ denotes a potential with the phasonic displacements $v$ and $\boldsymbol{w}$. For $j=0, \ldots, 13$, we obtain as suitable combinations

$$
\begin{aligned}
& \Delta \boldsymbol{u}=\left(u_{r} \cos (j \pi / 7), u_{r} \sin (j \pi / 7)\right), \\
& \Delta \boldsymbol{v}=\left(v_{r} \cos (3 j \pi / 7), v_{r} \sin (3 j \pi / 7)\right), \text { and } \\
& \Delta \boldsymbol{w}=\left(w_{r} \cos (5 j \pi / 7), w_{r} \sin (5 j \pi / 7)\right) .
\end{aligned}
$$

These displacements do not modify the potential when

$$
\begin{aligned}
& u_{r} / a_{\mathrm{V}}=\frac{8}{49}\left(n_{1} a+n_{2} b+n_{3} c\right), \\
& v_{r} / a_{\mathrm{V}}=\frac{8}{49}\left(n_{2} a+n_{3} b+n_{1} c\right), \\
& w_{r} / a_{\mathrm{V}}=\frac{8}{49}\left(n_{3} a+n_{1} b+n_{2} c\right),
\end{aligned}
$$

where $n_{1}, n_{2}$ and $n_{3} \in \mathbb{Z}$ and $a, b$ and $c$ are the constant values

$$
\begin{aligned}
& a=(1-\cos (10 \pi / 7))^{2}-(1-\cos (2 \pi / 7))(1-\cos (6 \pi / 7)), \\
& b=(1-\cos (6 \pi / 7))^{2}-(1-\cos (2 \pi / 7))(1-\cos (10 \pi / 7)), \\
& c=(1-\cos (2 \pi / 7))^{2}-(1-\cos (6 \pi / 7))(1-\cos (10 \pi / 7)) .
\end{aligned}
$$

Thus, a colloid at position $\boldsymbol{r}$ in a potential with phasonic displacements $\boldsymbol{v}$ and $\boldsymbol{w}$ can be mapped to a reduced position $\boldsymbol{r}^{(\mathrm{red})}=\boldsymbol{r}-\Delta \boldsymbol{u}$ in a potential with reduced phasonic displacements $\boldsymbol{v}^{(\mathrm{red})}=\boldsymbol{v}+\Delta \boldsymbol{v}$ and $\boldsymbol{w}^{(\mathrm{red})}=\boldsymbol{w}+\Delta \boldsymbol{w}$ inside the particular characteristic area.

To determine the size of the characteristic areas we consider the subspaces spanned by a chosen phononic direction $\boldsymbol{e}_{j}=(\cos (\pi j / 7), \sin (\pi j / 7))$ and its phasonic counterparts. Thus, we get a three-dimensional subspace in every direction. We have to find cuboids of the side lengths $\delta u, \delta v$, and $\delta w$ that fill out the whole volume when shifted about $\Delta u$ in phononic and $\Delta v$ and $\Delta w$ in phasonic directions. Then all reduced positions and reduced phasonic displacements are limited by

$$
\begin{aligned}
& \left|\boldsymbol{r}^{(\mathrm{red})} \cdot \boldsymbol{e}_{j}\right| \leq \delta u / 2 a_{\mathrm{V}}, \quad\left|\boldsymbol{v}^{(\mathrm{red})} \cdot \boldsymbol{e}_{j}\right| \leq \delta v / 2 a_{\mathrm{V}}, \\
& \text { and }\left|\boldsymbol{w}^{(\mathrm{red})} \cdot \boldsymbol{e}_{j}\right| \leq \delta w / 2 a_{\mathrm{V}}
\end{aligned}
$$

for all symmetry directions $j=0, \ldots, 13$. A possible choice is $\delta u / 2 a_{\mathrm{V}}=\delta v / 2 a_{\mathrm{V}}=\delta w / 2 a_{\mathrm{V}}=1 / 2(-a-b)$.

\section{Analyzing colloidal trajectories}

\subsection{Method}

In order to describe the colloidal trajectories in a quasicrystal with 14-fold symmetry, we have to consider a 4-dimensional phasonic space spanned by $v_{x}, v_{y}, w_{x}$, and $w_{y}$. Since it is too complex to sample the complete space, we restrict our analysis to drifts on selected planes in the phasonic space.

As a first attempt either $\boldsymbol{w}$ is varied while $\boldsymbol{v}=\mathbf{0}$ or $\boldsymbol{v}$ is changed while $\boldsymbol{w}=\mathbf{0}$. We trace the paths of colloids started at the origin for all drift directions within the respective phasonic plane. Then the important points of the trajectories for reduced phasonic displacements $\boldsymbol{w}^{(\mathrm{red})}$ or $\boldsymbol{v}^{\text {(red) }}$ and the corresponding reduced positions $\boldsymbol{r}^{\text {(red) }}$ can be presented in the diagrams of Fig. 2.

The diagrams correspond to those obtained for the $D=4$ quasicrystals in $[16,20]$. The procedure to derive a trajectory for the case $\boldsymbol{v}=\mathbf{0}$ (see Fig. 2a,b) is as follows: When the phasonic displacement $\boldsymbol{w}^{(\mathrm{red})}$ reaches 
(a)
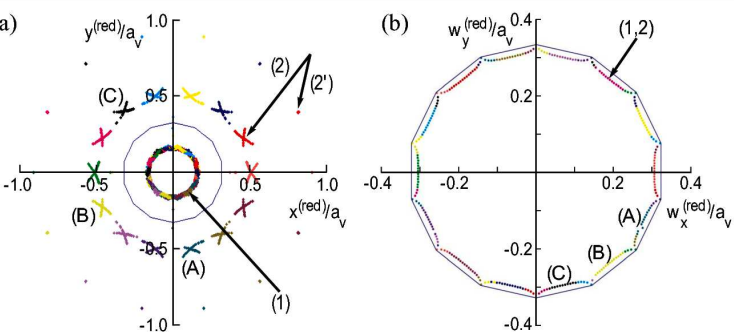

(c)
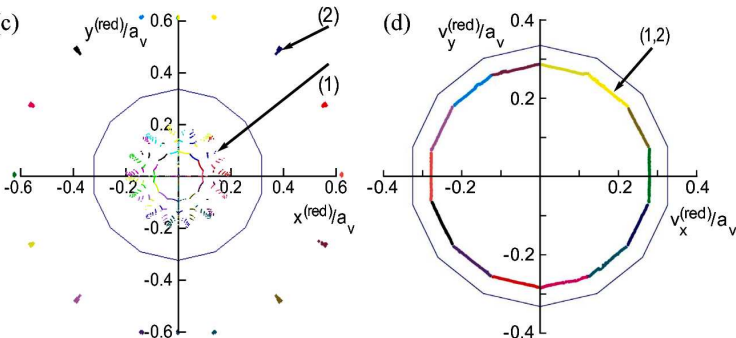

Fig. 2. (a,b) Areas of reduced positions $\boldsymbol{r}^{(\mathrm{red})}$ and reduced phasonic displacements $\boldsymbol{w}^{\text {(red) }}$ for $\boldsymbol{v}=\mathbf{0}$. (c,d) Areas of reduced positions $\boldsymbol{r}^{\text {(red) }}$ and reduced phasonic displacements $\boldsymbol{v}^{\text {(red) }}$ for $\boldsymbol{w}=\mathbf{0}$. The characteristic areas are limited by blue polygons. The lines where particle start to slide or end up after sliding or remapping are marked by different colors. Selected lines are also labelled by letters $(A),(B)$, or $(C)$ in order to denote corresponding lines.

a point marked by $(1,2)$ the colloid jumps from its old position (1) to a new one (2) of the same color. Corresponding colors of selected lines are also denoted by $(A)$, $(B)$, and $(C)$. Let us note that the positions $\left(2^{\prime}\right)$ are only reached for a drift along a symmetry axis or slightly beside it. Since the colloidal position is now outside the characteristic area, one has to map it back inside by employing combinations of $\Delta \boldsymbol{u}, \Delta \boldsymbol{w}$ and also $\Delta \boldsymbol{v}$. Due to the latter, for the mapped quantity $\boldsymbol{v}^{\text {(red) }} \neq \mathbf{0}$. Thus, one has to consider a new diagram for the reduced phasonic displacements $\boldsymbol{w}^{\text {(red) }}$ and the reduced positions $\boldsymbol{r}^{\text {(red) }}$ after every jump. The same difficulty arises for drifts in the $\boldsymbol{w}=\mathbf{0}$ plane. Therefore, in contrast to quasicrystals with rank $D=4$, the derivation of a colloidal trajectory for an arbitrary drift is for $D=6$ in most cases quite complex, as many diagrams would be required for a complete prediction.

\subsection{Selected trajectories}

There are drifts for which it is possible to deduce the trajectories with a single diagram for the reduced phasonic displacements. For certain drifts in the plane with $v_{y}=w_{y}=0$, the colloid moves in $x$ direction. In addition, the phasonic counterparts $\Delta \boldsymbol{w}$ and $\Delta \boldsymbol{v}$ to the required $\Delta \boldsymbol{u}$ only affect the $x$ components $w_{x}$ and $v_{x}$. As a consequence, the phasonic plane with $v_{y}^{(\text {red })}=w_{y}^{(\text {red })}=0$ is never left during the mapping process. In the following, we discuss for selected drifts the colloidal trajectories that can be completely derived from the diagram in Fig. 3a. (a)
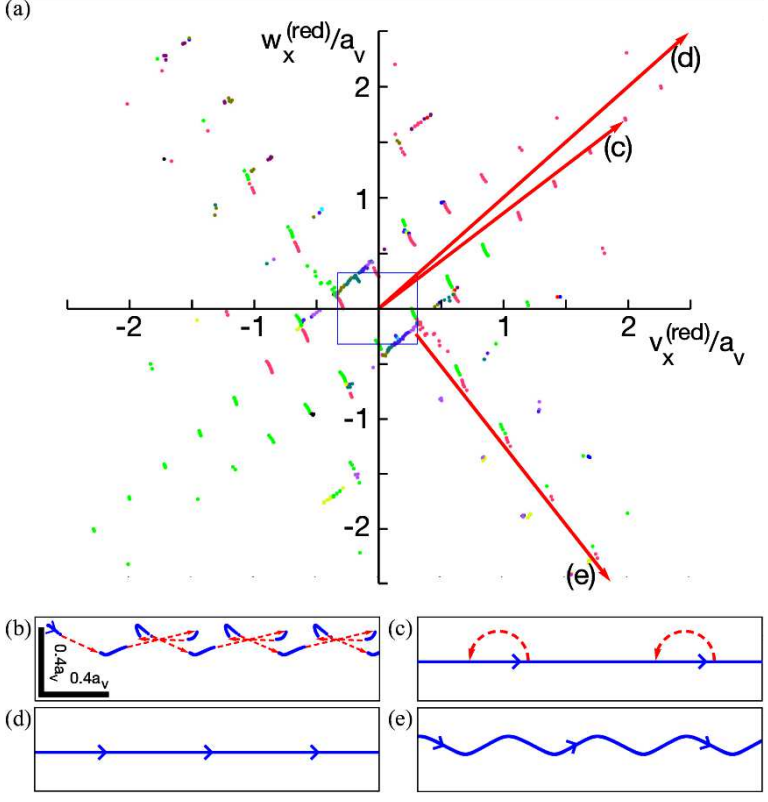

Fig. 3. (a) Section of the characteristic diagram for the reduced phasonic displacements $v_{x}^{(\text {red })}$ and $w_{x}^{(\text {red })}$ with $v_{y}=w_{y}=0$. (b)-(e) Selected typical colloidal trajectories arising for $v_{x}-w_{x}$ drifts in certain directions. The directions of motion are indicated by arrows. The scale bar denotes the length scales in $x$ - and $y$-direction.

For a phasonic drift in the direction $\left(v_{x}, v_{y}, w_{x}, w_{y}\right)=$ $(\cos (3 \pi / 7), 0, \cos (5 \pi / 7), 0)$ we find a zigzag path illustrated in Fig. 3b. It is similar to the zigzag paths in quasicrystals with rank $D=4$ which is why we refer for a detailed description to [16].

On the other hand, a new type of trajectory is depicted in Fig. 3c. It consists of straight paths interrupted by jumps in the opposite direction and occurs when a drift in the direction $\left(v_{x}, v_{y}, w_{x}, w_{y}\right)=$ $(\cos (9 \pi / 70), 0, \cos (3 \pi / 14), 0)$ is applied. The particle moves on a straight line until the phasonic displacement reaches a point in the diagram in Fig. 3a such that the particle jumps. The phasonic displacements and the positions can be mapped back into the characteristic areas to predict the further path of the colloids when the displacement is further increased.

Figure 3d illustrates an example of a straight path without any jumps. It arises for a phasonic drift in the direction $\left(v_{x}, v_{y}, w_{x}, w_{y}\right)=(1,0,1,0)$. In this case, the phasonic displacement never reaches a point in the diagram Fig. 3a and as a consequence the particle never leaves a minimum. Therefore, the phasonic displacement is simply mapped back when it crosses the border of its characteristic area. This drift proceeds along a symmetry plane in the phasonic space such that the form of the trajectory is a straight line.

Another new type of trajectory has the shape of a winding line as shown in Fig. 3e. It arises, e.g., when a drift in the direction $\left(v_{x}, v_{y}, w_{x}, w_{y}\right)=$ 
$(\cos (3 \pi / 7), 0, \cos (5 \pi / 7), 0)$ is applied for a colloid position whose initial reduced phasonic displacement is not in the origin but at $v_{x}=0.3 a_{\mathrm{V}}$ and $w_{x}=-0.2 a_{\mathrm{V}}$. Just as in the example before, the colloid never jumps. Compared to the previous case the drift does not move along a symmetry plane in the phasonic space which leads to the occurrence of curves in the path.

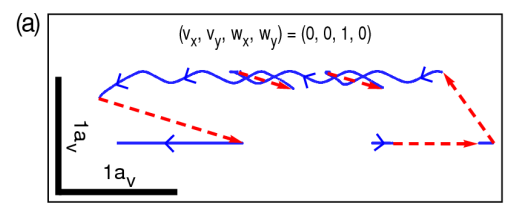

(b)

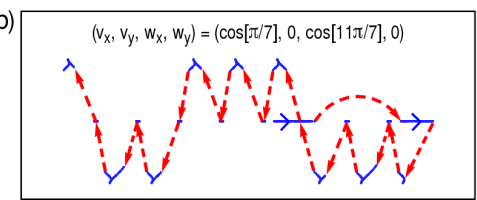

Fig. 4. General colloidal trajectories representing the majority of the observed colloidal paths: (a) A combination of straight and winding lines separated by jumps. (b) Several jumps along an irregular zigzag path. (c) Chaotic trajectory consisting of irregular slides and jumps in between.

Beside these presented typical paths, for most drifts we find much more complex trajectories. Further examples are shown in Fig. 4. Let us note that all paths of the colloids are composed of the typical trajectories such that every section can be explained separately.

\section{Conclusions}

On the basis of the 14-fold rotational symmetry, we have extended previous studies $[16,20]$ to quasicrystals with rank $D=6$. We showed how one can still predict colloidal trajectories that are caused by phasonic drifts in spite of the increased complexity in the phasonic space. In addition, we were able to identify new types of trajectories in addition to the regular straight and zigzag paths that are already known from quasicrystals with rank $D=4$

\section{Acknowledgments}

We thank J. Kromer, J. Roth, and H. Stark for helpful discussions and the Deutsche Forschungsgemeinschaft for financial support (Schm 2657/2 and Ro 924/5).

\section{References}

[1] R. Lifshitz, Found. Phys. 33, 1703 (2003).

[2] C.L. Henley, M. de Boissieu, W. Steurer, Phil. Mag. 86, 1131 (2006).

[3] H. Löwen, J. Phys.: Cond. Matt. 13, R415 (2001).

[4] A. Ashkin, Phys. Rev. Lett. 24, 156 (1970).

[5] A. Ashkin, Science 210, 1081 (1980).

[6] M.M. Burns, J.-M. Fournier, J.A. Golovchenko, Science 249, 749 (1990).

[7] M. Schmiedeberg, J. Roth, H. Stark, Eur. Phys. J. E 24, 367 (2007).

[8] J. Mikhael, J. Roth, L. Helden, C. Bechinger, Nature 454, 501 (2008).

[9] M. Schmiedeberg, H. Stark, Phys. Rev. Lett. 101, 218302 (2008).

[10] J. Mikhael, M. Schmiedeberg, S. Rausch, J. Roth, H. Stark, C. Bechinger, Proc. Nat. Acad. Sci. 107, 7214 (2010).

[11] M. Schmiedeberg, J. Mikhael, S. Rausch, J. Roth, L. Helden, C. Bechinger, H. Stark, Eur. Phys. J. E 32, 25 (2010).

[12] J. Mikhael, G. Gera, T. Bohlein, C. Bechinger, Soft Matter 7, 1352 (2011).

[13] C. Reichhardt, C.J. Olson Reichhardt, Phys. Rev. Lett. 106, 060603 (2011).

[14] P. Kählitz, M. Schoen, H. Stark, J. Chem. Phys. 137, 224705 (2012).

[15] P. Kählitz, H. Stark, J. Chem. Phys. 136, 174705 (2012).

[16] J.A. Kromer, M. Schmiedeberg, J. Roth, H. Stark, Phys. Rev. Lett. 108, 218301 (2012).

[17] T. Bohlein, C. Bechinger, Phys. Rev. Lett. 109 058301 (2012).

[18] M. Schmiedeberg, H. Stark, J. Phys.: Cond. Matt. 24, 284101 (2012)

[19] T. Dotera, Isr. J. Chem. 51, 1197 (2011).

[20] M. Sandbrink, M. Schmiedeberg, in: Aperiodic Crystals, Eds. S. Schmid, R.L. Withers, R. Lifshitz, Springer, Berlin 2013, p. 261.

[21] S. Gorkhali, J. Qi, G. Crawford, J. Opt. Soc. Am. B 23, 149 (2006).

[22] D. Levine, T.C. Lubensky, S. Ostlund, S. Ramaswamy, P.J. Steinhardt, J. Toner, Phys. Rev. Lett. 54, 1520 (1985)

[23] J.E.S. Socolar, T.C. Lubensky, P.J. Steinhardt, Phys Rev. B 34, 3345 (1986).

[24] R. Lifshitz, Z. Kristallogr. 222, 313 (2007).

[25] R. Lifshitz, Isr. J. Chem. 51, 1156 (2011). 